\title{
An Approach for Estimating the Deformation of Pulsar Included in Binary-System PSR B 1913+16
}

\author{
M. H. M. Soleiman", M. M. H. El Gogary*, S. S. Abdel-Aziz", and A. I. Imsaeri",** \\ "Physics Department, Faculty of Science, Cairo University, Giza 12613, Egypt. \\ ** Physics Department, Faculty of Science, Sebha University, Sebha, Libya.
}

\begin{abstract}
$\mathbf{D}^{1}$ ISCOVERY of pulsars included in binary systems (BS) is dated to 1974, when Hulse and Taylor announced the parameters of pulsar PSR B 1913+16.It inspires great motivations for possible new physical measurements of the properties of neutron stars (NSs). We present a simple model to estimate the deformation in shape of two neutron stars bounded to each other in a binary system due to their mutual gravity. The model predicts the Bulk modulus of the pulsar - as an infinite nuclear matter. In particular, the model determines the stress and the strain of a pulsar included in PSR B 1913+16 binary system within the frame of Newtonian theory of gravity. The calculations show that, our approach is consistent with the model of two spherical neutron stars up to the eccentricity-squared. The model could evaluate the axial elongation of the observed NS as well as the eccentricity. The eccentricity is found to be of order $10^{-5}$ and the elongation is of the order $10^{-9} \mathrm{~km}$.
\end{abstract}

Keywords: Infinite Nuclear Matter, Neutron star, Pulsar, Bulk modulus, Compressibility, Companion star.

\section{Introduction}

Pulsars PSR B1913+16 (PSR J1915+1606) and PSR B 1534+12 would exhibit a rich set of potentially measurable relativistic effects. Subsequent studies have shown that the pulsar's companion must also be a neutron star [1, 2, 3]. The systems are essentially clean laboratories for testing relativistic gravity. The tidal and rotational stellar distortions are complicating factors for any dynamical analysis and the largest remaining complications depend on referenceframe accelerations related to the structure and dynamics of our Galaxy.

Deformation of neutron stars is controlled by the equation of state (EoS) of the neutron stars. The difficulty in estimating the neutron star deformation parameters is coming from the absence of physical models relating stress and strain on the matter of the neutron star. Using EoSs, several studies estimate the compressibility coefficients of the neutron stare to be in the range 180-240 MeV for finite heavy nuclei by performing effective interactions-microscopic calculations [4]. It is found that some effective Lagrangians having a bulk compression modulus in the range $280-350 \mathrm{MeV}$ can predict correctly breathing mode energies in medium and heavy nuclei [5]. A symmetric matter incompressibility parameter $K_{S}=240 \mathrm{MeV}$ fits the heavy-ion data [6]. Using a quantum hydrodynamic effective model in the mean-field approximation to the description of neutron stars, it could be predicted that, the compression modulus of nuclear matter is $K=257.2 \mathrm{MeV}$ [7].

It is possible to estimate the strain and stress on the surface of a pulsar included in a binary system in the frame of Newtonian dynamics. Horowitz and Kai Kadau's [8] published simulations of Coulomb solids for representing neutron star crust show that,the breaking strength of neutron star crust is about $10^{10}$ times more than for terrestrial engineering materials such as metal-alloys where the strength is measured in fractions of a GPa, and the breaking strain is about 0.1. A stable and unique value of the infinite nuclear matter compression modulus $\left(\mathrm{K} \_\infty=288 \pm 20 \mathrm{MeV}\right)$ has been extracted using the masses of all known nuclei $[9,10]$.The extraction method does not use any effective interaction or dynamic property like monopole resonance.

In the present work, the gravitational effect on the deformation of a pulsar included in a binary system will be modeled in order to calculate the potentials and the force components in the body 
and on the surface of the pulsar. Section 1 will exhibit the basic model of a pulsar in a binary system. Section 2 shows the developed model to define the deformation of the pulsar using the terminology of ref. [11]. Section 3 are the results and discussions. Finally, section 4 is left for the conclusions.

\section{Basic Parameters of PSR B 1913+16}

The $1^{\text {st }}$ binary pulsar system PSR B1913+16 was discovered by Hulseand Taylorin summer,1974 [12] in a systematic search for new pulsars. The nominal pulse period of the pulsar is $59 \mathrm{~ms}$. This short period was observed to be periodically shifted (Doppler Shift), which proves that the pulsar is a member of a binary system with an orbital period of 7.75 hours. With Kepler's third law and reasonable masses, one concludes from this that the system is rather narrow, having a diameter of roughly $1 \mathbf{R}_{\odot \text { correspondingly, }}$ the velocity of the pulsar $\sim 10^{-3} c$ and it moves through a relatively strong gravitational field ( $\left.G M / C_{C^{2} r} \sim 10^{-6}\right)$. These parameters show that several special and general relativistic effects should be observable. This calculations have been achieved over three decades with increasing accuracy [13]. The measurements on the system PSR B1913+16 yield enough information to determine all parameters of the system as shown in Table 1, and allow in addition a test of general relativity (GR)-results, provided that; the companion star has a negligible quadrupole moment and tidal interactions are small enough [14]. These conditions for a "clean" relativistic system are probably fulfilled.

\section{The model}

The proposed model aims to setup a calculation schem of the deformation of a neutron star included in a binary system. The basic assumbtions of the model are;

1. The deformasion of the absorved NS is due to the resultant forces of the Newtonian-gravition.

2. The intial shape of NS is a sphere at apastron, and after the deformation at the preiastron, the shape will be an ellipsoid of revolution with small eccentricity $e$ about z-axis. The deformation is along the z-axis, preserves the radius in XY-plane, and occures according to the classical tide-theory [15].

3. NS is, instantly, in thermodynamic equilib- rium (static NS,) and the mechanical balance is between the internal pressure force and the resultant gravitional force.

4. The spinning of NS about the z-axis will not affect the deformation of the NS, specifically for symmetric deformation.

5. The density of NS is uniform and denoted by $\rho_{N S}$.

The proposed model of the pulsar and a companion NS included in binary system and orbitting about their common center of mass is illustrated in Fig. 1.

The effect of the companion (NS) on deformation of the observed pulsar is shown in Fig. 2. The pulsar orbital motion includes an acceleration toward the companion (NS). This acceleration affects any surface element on pulsar, as it orbits with the companion NS. The resulting deformation corresponds to the pull at the pulsar's center. Any surface elements on the companion (NS) side of the pulsar (point B) is pulled by the companion (NS) with a force that is slightly greater than it would be at the center. The surface of the pulsar which is nearest side to the companion (NS) behaves as if they suffer a slight additional attraction toward the companion (NS). On the other side the surface on the far side from the companion (NS) (point A) are pulled by the companion (NS) with a force that is slightly weaker than it would be at the center. Also it is clearfrom the Fig. 2, that, at the points $\mathrm{P}$ and $\mathrm{Q}$ there is weaker deformation effects than at points A and B because of small compressibility of Neutron star matter. It is possible to reason why must the deformations of the nearest and the farthest parts of the pulsar be symmetric? The answer is that, the acceleration $a$ of an element $\Delta m$ on the pulsar surface is evaluated from Newton's laws followedby Tailor-expansion for the variable $\left(d=d_{0} \mp R\right)$ up to order one about $d_{\mathbf{0}}$ (the z-coordinates of the center of mass),we get $a \approx-\frac{G M}{d_{\mathbf{0}}} \pm \frac{G M}{d_{\mathbf{0}}^{2}} R=-\frac{G M}{\left(d_{0}^{2}\right)\left(d_{0} \mp R\right)}$. That is, at any instance of observation time the relative velocities of the surface elements cause a type of symmetric instantaneous deformations of the whole body of the pulsar. The instantaneous deformations reshape the spherical NS to an ellipsoid of revolution with the major axis is along the line passing through the centers of the two NSs. 
TABLE 1. The classical orbital elements, astrometric parametersand post Keplerian parameters for the most famous relativistic binary system PSR B1913+16 taken from reference [14].

\begin{tabular}{|c|c|c|}
\hline & Parameters & \\
\hline Classical orbital elements & $\begin{array}{l}\text { Orbital period } p_{b}[d] \\
\text { Eccentricity } \\
\text { Semi major axis } a_{p} \sin i / c[s] \\
\text { Periastron length } \omega_{0}[\mathrm{deg}] \\
\text { Periastron passage } T_{0}[\mathrm{MJD}]\end{array}$ & $\begin{array}{l}0.322997462 \\
0.6171308(4) \\
2.3417592(19) \\
226.57528(6) \\
46443.99588319(3)\end{array}$ \\
\hline Astrometric parameters & $\begin{array}{l}\text { Spin period } P[\mathrm{~ms}] \\
\text { Braking rate } \dot{P}\left[10^{-18}\right] \\
\text { Right ascension }(\mathrm{J} 2000) \\
\text { Declination }(\mathrm{J} 2000) \\
\text { Dispersion }\left[p c \mathrm{~cm}^{-3}\right] \\
\text { Timing accuracy }[\mu s]\end{array}$ & $\begin{array}{l}\text { 59.029997929613(7) } \\
8.62713(8) \\
\text { 19: } 15: 28.0002 \\
\text { 16: } 06: 27.4043 \\
168.770 \\
15\end{array}$ \\
\hline Post Keplerian parameters & $\begin{array}{l}\text { Periastron shift } \omega^{\circ}[\mathrm{deg} / \mathrm{yr}] \\
\text { Grav/Doppler effect } \gamma R D[\mathrm{~ms}] \\
\text { Shapiro time delay } r[\mu s] \\
\text { Orbital inclination } s=\sin i \\
\text { Orbit decay } \dot{P}_{b}\left[10^{-12} s / \mathrm{s}\right]\end{array}$ & $\begin{array}{l}4.226621(11) \\
4.295(2) \\
- \\
-2.422(6)\end{array}$ \\
\hline
\end{tabular}

The gravitational potential $U(r)$ is calculated analytically inside and outside an ellipsoid and sphere by integration over the mass-element $d m^{\prime}=\rho_{N S} d V^{\prime}$ of a single NS using Newton's law of gravitation potential.

$$
U(r)=-G \int_{N S} \frac{\rho_{N S}}{\left|\vec{r}-\overrightarrow{r^{\prime}}\right|} d V^{\prime}, \quad d V^{\prime}=\rho^{\prime} d \rho^{\prime} d_{\phi^{\prime} d z^{\prime}}
$$

Where

$$
\rho^{\prime} \in\left[0, f_{s}\left(z^{\prime}\right)\right], \quad f_{s}\left(z^{\prime}\right)= \begin{cases}+R \sqrt{1-\left(\mathbf{z}^{\prime} / \mathbf{R}\right)^{2}} & \text { for a sphere, } \\ +R \sqrt{1-\left(z^{\prime} / \tilde{R}\right)^{2}} & \text { for an ellipsoide }\end{cases}
$$




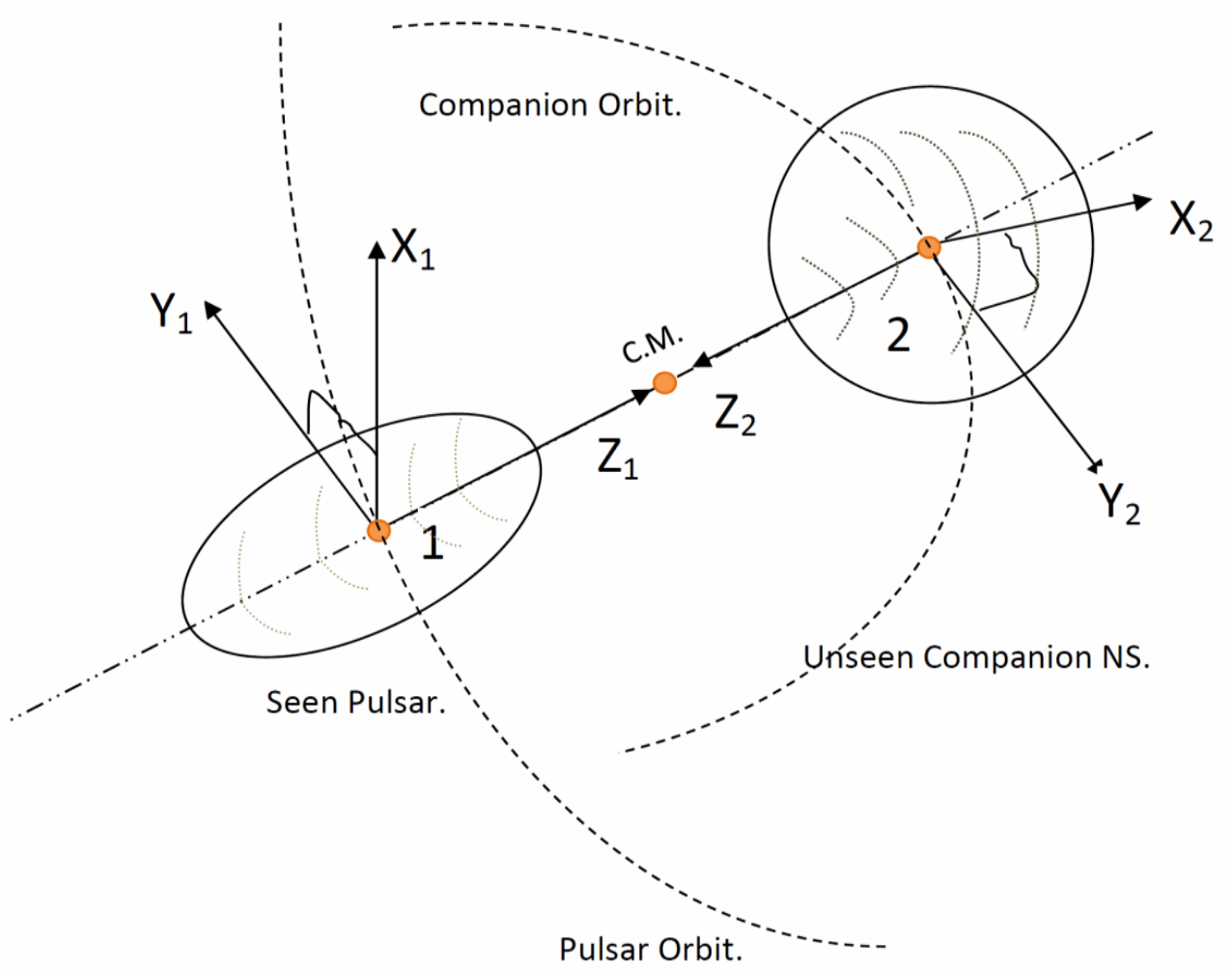

Fig. 1. Illustration by the authors of the binary system of two NSs are orbiting each other about their common center of mass (C. M.) The observed pulsar is deformed to an ellipsoid (= a body of revolution). The illustration represents the coordinate system of each NS.

$$
\begin{aligned}
& \rho^{\prime} \in\left[0, f_{S}\left(z^{\prime}\right)\right], \quad f_{S}\left(z^{\prime}\right)=\left\{\begin{array}{l}
+R \sqrt{1-\left(\mathbf{z}^{\prime} / \mathbf{R}\right)^{2}} \\
+R \sqrt{\mathbf{1}-\left(z^{\prime} / \widetilde{R}\right)^{2}}
\end{array} \quad\right. \text { for a sphere, } \\
& \widetilde{R}=\frac{R}{\sqrt{1-e^{2}}} \text {, for the spherical shape. }
\end{aligned}
$$

$$
\begin{aligned}
& \left|\vec{r}-\overrightarrow{r^{\prime}}\right|=\sqrt{\left(\rho \cos \phi-\rho^{\prime} \cos \phi^{\prime}\right)^{2}+\left(\rho \sin \phi-\rho^{\prime} \sin \phi^{\prime}\right)^{2}+\left(z-z^{\prime}\right)^{2}} \\
& =\sqrt{\rho^{2}+\rho^{\prime 2}-2 \rho \rho^{\prime} \cos \left(\phi-\phi^{\prime}\right)+\left(z-z^{\prime}\right)^{2}}
\end{aligned}
$$

Along the z-axis .

$$
\left|\vec{r}-\overrightarrow{r^{\prime}}\right|=\sqrt{\ldots}\left(\rho^{\prime 2}+\left(z-z^{\prime}\right)^{2}\right)
$$




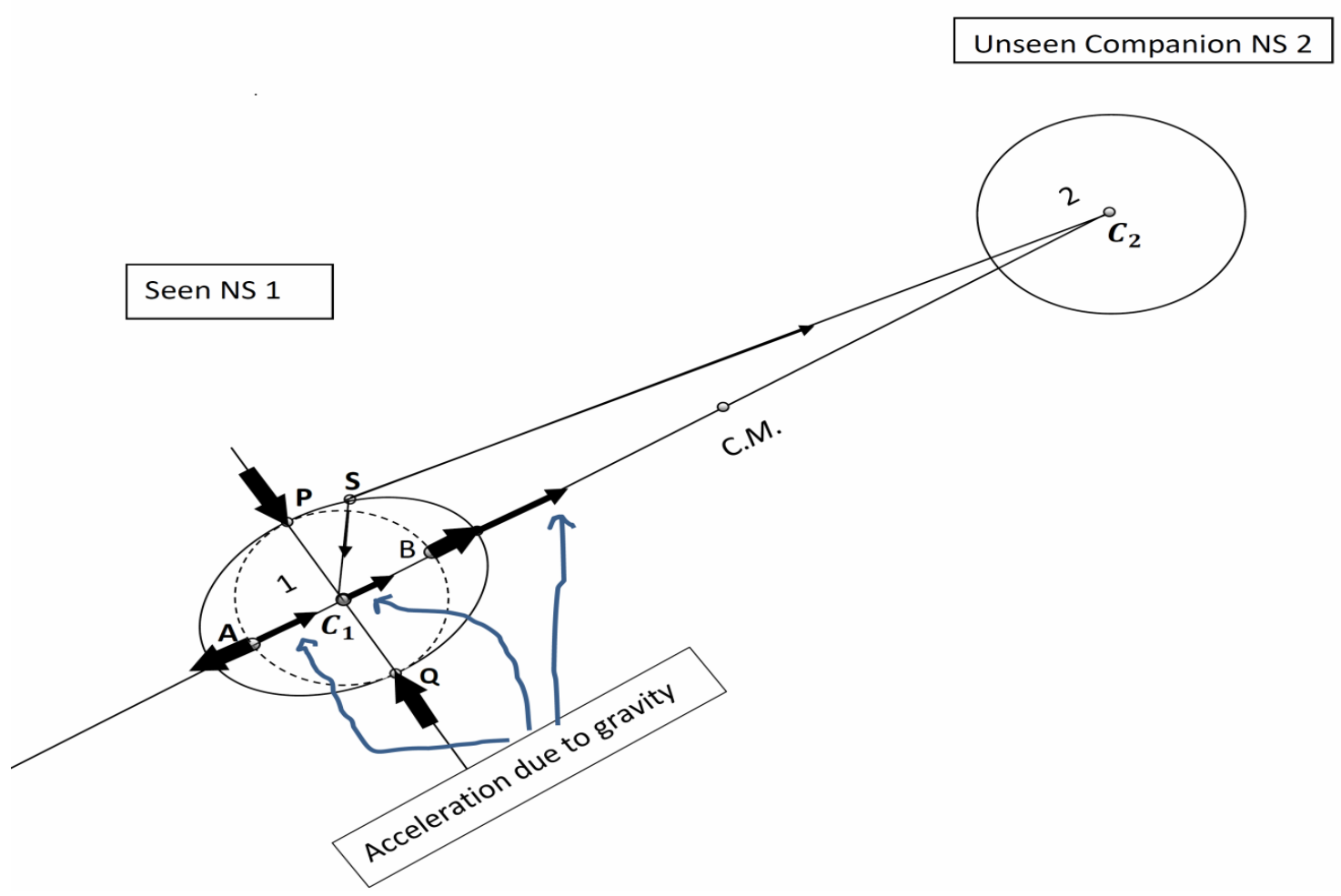

Fig. 2. Illustration by the authors for the binary system, where the observed NS (the pulsar) is deformed according to the tide-theory [15]. The heavy arrows represent the instantenuous symmetric deformations due to different accelerations of the body-elements as seen in the extrinsic coordinatesof the pulsar due to the companion NS.

Performing the integration procedeure for $\rho^{\prime} d \rho^{\prime}$, and then obtain the series expantion up to order $e^{2}$. The integration for $\left(\phi^{\prime}\right)$ is cyclic due to the assumed geometrical symmetry. The last integeration for $\left(Z^{\prime}\right)$ is done analytically for each series term up to order $e^{2}$. Finally the potentials inside $(|Z| \leq \widetilde{R})$ and outside $(|Z|>\widetilde{R})$ the ellipsoid $(\mathrm{Ei}, \mathrm{Eo})$ and the sphere $(\mathrm{Si}, \mathrm{So})$ could be written as;

$$
\begin{aligned}
& \mathbf{U}^{\mathrm{Ei}}(z ; R, e)=\frac{2}{3} \pi \mathbf{G} \boldsymbol{\rho}_{\mathbf{N S}}\left(-3 R^{2}+z^{2}\right)-\mathbf{e}^{2} \frac{2}{15} \pi \mathbf{G} \boldsymbol{\rho}_{\mathbf{N S}}\left(5 R^{2}+2 z^{2}\right)+o\left(e^{4}\right) \\
& \mathbf{U}^{\mathrm{Eo}}(z ; R, e)=-\frac{4 \pi \mathbf{G} \boldsymbol{\rho}_{\mathbf{N S}} R^{3}}{3 z}-\mathbf{e}^{2} \frac{2 \pi \mathbf{G} \boldsymbol{\rho}_{\mathbf{N S}}\left(2 R^{5}+5 R^{3} z^{2}\right)}{15 z^{3}}+o\left(e^{4}\right) \\
& \mathbf{U}^{S i}(Z ; R)=\frac{2}{3} \pi \mathbf{G} \boldsymbol{\rho}_{\mathbf{N S}}\left(-3 R^{2}+z^{2}\right) \\
& \mathbf{U}^{\text {So }}(Z ; R)=-\frac{4 \pi \mathbf{G}_{\mathbf{N S}} R^{3}}{3 z} .
\end{aligned}
$$


The integrant in equation (1) includes singularity at for the case $|Z| \leq \widetilde{R}$. This singularity point requires mathematical labor for performing the integration over $z^{\prime}$.

To find the stress, first obtain the negative of the gradient of the Gaussian potential inside (i) and outside (o) the ellipsoid (E) and the sphere $(\mathrm{S})$ to get the z-component of the forces per unit mass: $\mathbf{F}_{z}^{\mathbf{E i}}(z), \mathbf{F}_{z}^{\mathbf{E o}}(z), \mathbf{F}_{z}^{\mathbf{S i}}(z)$, and $\mathbf{F}_{z}^{\text {So }}(z)$ respectively. They are calculated analytically and found to be:

$$
\begin{aligned}
& \mathbf{F}_{Z}^{\mathrm{Ei}}(z)=\frac{\mathbf{4}}{\mathbf{1 5}} \pi \mathbf{G} \boldsymbol{\rho}_{\mathbf{N S}}\left(-\mathbf{5}+2 \mathbf{e}^{2}\right)_{z} . \\
& \mathbf{F}_{Z}^{\mathrm{Eo}}(z)=-\frac{2 \pi \mathbf{G} \boldsymbol{\rho}_{\mathbf{N S}} \pi R^{3}\left(6 \mathbf{e}^{2} R^{2}+\mathbf{5}\left(2+\mathbf{e}^{2}\right) z^{2}\right)}{15 z^{4}} . \\
& \mathbf{F}_{Z}^{\mathbf{S i}}(z)=-\frac{\mathbf{4}}{\mathbf{3}} \pi \mathbf{G} \boldsymbol{\rho}_{\mathbf{N S}} z . \\
& \mathbf{F}_{Z}^{\mathbf{S o}}(z)=-\frac{\mathbf{4} \pi \mathbf{G} \boldsymbol{\rho}_{\mathbf{N S}} R^{3}}{\mathbf{3}^{2}} .
\end{aligned}
$$

Where $R$ is radius, $G$ is gravitation constant, $\rho_{\mathrm{Ns}}$ is density, and $e$ is eccentricity.

The z- component of the resultant of the forces per unit mass $\mathbf{F}_{Z}^{\operatorname{Res}}$ on the mass element $d m=d A_{\perp} d z \rho_{\mathbf{N S}}(z)$ at the surface of the observed NS is found by:

$$
\begin{aligned}
& \mathbf{F}_{Z}^{\text {ERes }}=\mathbf{F}_{Z}^{\mathbf{E i}}\left(R_{\mathbf{1}}^{\sim}\right)-\mathbf{F}_{Z}^{\text {Eo }}\left(d_{\text {Peria }}-\tilde{R}_{\mathbf{1}}\right) \\
& \mathbf{F}_{Z}^{\text {SRes }}=\mathbf{F}_{Z}^{\text {Si }}\left(R_{\mathbf{1}}\right)-\mathbf{F}_{Z}^{\text {So }}\left(d_{\text {Apas }}-R_{1}\right)
\end{aligned}
$$

Where $d_{\text {Peria and }} d_{\text {Apas }}$ are the distances between the centers of the two neutron stars at periastron and apstron, respectively. $R_{1}$ is the radius of the observed NS, when it was a sphere.

$$
\widetilde{R}_{1}=\frac{R_{1}}{\sqrt{1-e^{2}}} \approx R_{1}\left(1+\frac{1}{2} e^{2}\right)
$$

Then the stress $\left(\sigma_{z z}\right)$ can be written as,

$$
\sigma_{z Z}=\frac{d F_{Z}}{d A_{\perp}}=\frac{\left(\mathbf{F}_{Z}^{\text {ERes }}-\mathbf{F}_{Z}^{\text {SRes }}\right) d m}{d A_{\perp}}=\left(\mathbf{F}_{Z}^{\text {ERes }}-\mathbf{F}_{Z}^{\text {SRes }}\right) \boldsymbol{\rho}_{\mathrm{Ns}} \Delta z
$$

Egypt. J. Phys. Vol. 47 (2018)

$$
\sigma_{Z Z}=\frac{d F_{Z}}{d A_{\perp}}=\frac{\left(F_{Z}^{\text {ERes }}-F_{Z}^{\text {SRes }}\right) d m}{d A_{\perp}}=\left(\text { FER }_{Z}^{\text {ERes }} \text { F }_{Z}^{\text {SRes }}\right)_{\rho_{N S} \Delta z}
$$

The volume of ellipsoid and sphere are given respectively by,

$$
V_{\text {sphere }}=\frac{4}{3} \pi R_{1}^{3} V_{\text {sphere }}=\frac{4}{3} \pi R_{1}^{3},
$$

The strain given by,

$$
\epsilon=\frac{d V}{V}=\left(\frac{V_{\text {Ellipsoid }}-V_{\text {sphere }}}{V_{\text {sphere }}}\right)=\frac{\tilde{R}_{1}-R_{1}}{R_{1}} \underset{2}{2} z_{e}
$$

The elastic modulus of volume-deformation is known as the bulk modul and is defined by (BM) $=\frac{\text { stress }}{\text { strain }}$

The compressibility is a thermodynamic coefficient, defined by:

$$
\therefore \quad K=\frac{1}{B M}
$$

Then the compressibility can be written as,

$$
K=\frac{\text { strain }}{\text { stress }}=\frac{\left(R_{\mathbf{1}}-R_{\mathbf{1}}\right)}{R_{\mathbf{1}}\left(\mathbf{F}_{7}^{\text {ERes }}-\mathbf{F}_{7}^{\text {SRes }}\right) \mathbf{\rho}_{\mathrm{vs}} \Delta z}
$$

That is the compressibilty is a function of the NS-parameters $\left(R, \rho_{N S}, e\right)$. The published estimations of the compressibilty are coming from the proposed equations of state of the infinite nuclear matter $[16,17]$. Our model is successeded in estimating the value of the deformation along z-axis and give an acceptable picture of the effect of the gravitational interaction on the infinite nuclear matter. In future work the authors will introduce the modification to the model, which enable the astrophysists to estimate the deformation magnitude of a NS in BS. 


\section{$\underline{\text { Results and Discussions }}$}

The authors find Newtonian potentials and forces (see equations 4-6 for calculating potentials and equations 7-10 for calculating z-components of the forces) according to the proposed model for the observed pulsar as a

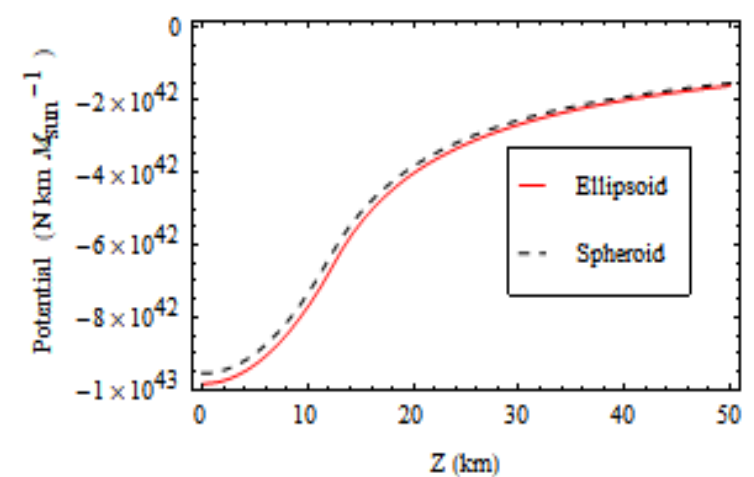

compact object included in a binary system. Figure 3a presents the comparison between the spherical and ellipsoidal single pulsar potentials and Figure $3 \mathrm{~b}$ is a comparison between forces, where the eccentricity and the radius of the pulsar are assumed to be $e=0.3, R_{\mathbf{1}}=12 \mathrm{~km}$.

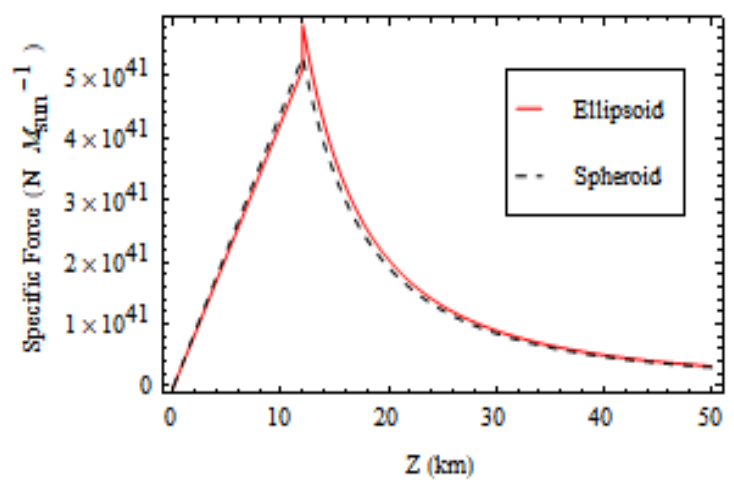

Fig. 3. The calculated gravitational potentials (a) and forces (b) for a single compact object (pulsar) as a function of the distance along z-axis. The dashed lines are the usual known Gaussian potentials and forces inside and outside spherical body $\left(R_{1}=12 \mathrm{~km}\right)$. The solid lines are the same calculation for ellipsoidal body $\left(R_{1}=12 \mathrm{~km}\right.$, and $\left.\mathrm{e}=\mathbf{0 . 3}\right)$.

The potentials in Fig. 3a have a characteristic and constant values at $Z=\mathbf{0}$. That are, $\mathbf{U}^{\mathrm{Ei}}\left(0 ; R_{1}, e\right)=-2 \pi \mathrm{G} \rho_{\mathrm{NS}} R_{1}^{2}-\mathrm{e}^{2} \frac{2}{3} \pi \mathrm{G} \rho_{\mathrm{NS}} R_{1}^{2}+o\left(e^{4}\right)$ , a n d $\mathbf{U}^{\mathbf{S i}}\left(\mathbf{0} ; R_{1}\right)=-\mathbf{2} \pi \mathbf{G} \boldsymbol{\rho}_{\mathbf{N} \mathbf{s}_{1}^{2}}$. T h e potential at the surface of neutron $\operatorname{star}\left(Z=R_{1}\right.$ ) is another chractaristic quantity. That are, $\mathrm{U}^{\mathrm{Ei}}\left(\widetilde{R_{1}} ; R_{1}, e\right)=-\frac{4}{3} \pi R_{1}^{2} \mathrm{G} \rho_{\mathrm{NS}}-\mathrm{e}^{2} \frac{8}{5} \pi R_{1}^{2} \mathrm{G}_{\mathrm{NS}}+O\left(e^{4}\right)$ ,and $\mathbf{U}^{\mathrm{Si}}\left(R_{1} ; R_{1}\right)=-\frac{\mathbf{4}}{\mathbf{3}} \pi R_{1}^{2} \mathrm{G} \boldsymbol{\rho}_{\mathrm{NS}}$. at $12 \mathrm{~km} 12 \mathrm{~km}$ in figure $3 \mathrm{~b}$ is due to the approximation $\widetilde{R_{\mathbf{1}}} \approx R_{\mathbf{1}}(1+1 / 2 e)$ through out the numerical calculation. The discontinuity in the curves of the force is due to the different functions describe the inside and the outside potentials which must coinside at the surface of the NS. The potential and the force of the ellipsoidal body resulting from the infinitisemal deformation of the spherical body are consistant and have similar chractaristics and behaviors as in the case of spherical bodies.

The compressibility $(K)$ is calculated for the proposed model according to equation (17) and shown in Fig. 4.
It is clear that, as the deformation increases the eccentricity increases and the compressibility decreases, i.e. the same gravitational forces could cause more deformation. That is the neutron star material becomes more soft.

The compressibility of finite nuclear matter in its ground state is calculated by using the highly symmetric skyrme potential of a shell model in the paper of M. M. Sharma [19]. The conclusion is that, the compressibility $K$ should be between $270-300 \mathrm{MeV}$ for the neutronpair separation energy to be consistent with the experimental data on the charge - radii.

M. M. Sharma had taken the saturation density to be in the range 0.160 to $0.170 \mathrm{fm}^{-3}$ , while the saturation binding energy is fixed at $-16.0 \mathrm{MeV}$ and the effective mass is froen at 0.79 . There is a strong correlation between the neutron-pair separation energy and the compressibility $K$.

The infinite nuclear matter could be studied through its equation of state. K. Hassaneen and H. Mansour in reference [18] had calculated the binding energy per nucleon (E/A) using a self-consistent Greens function method of the Brueckner-Hartree-Fock approach. Several values of the compressibility $K$ of infinite Egypt. J. Phys. Vol. 47(2018) 


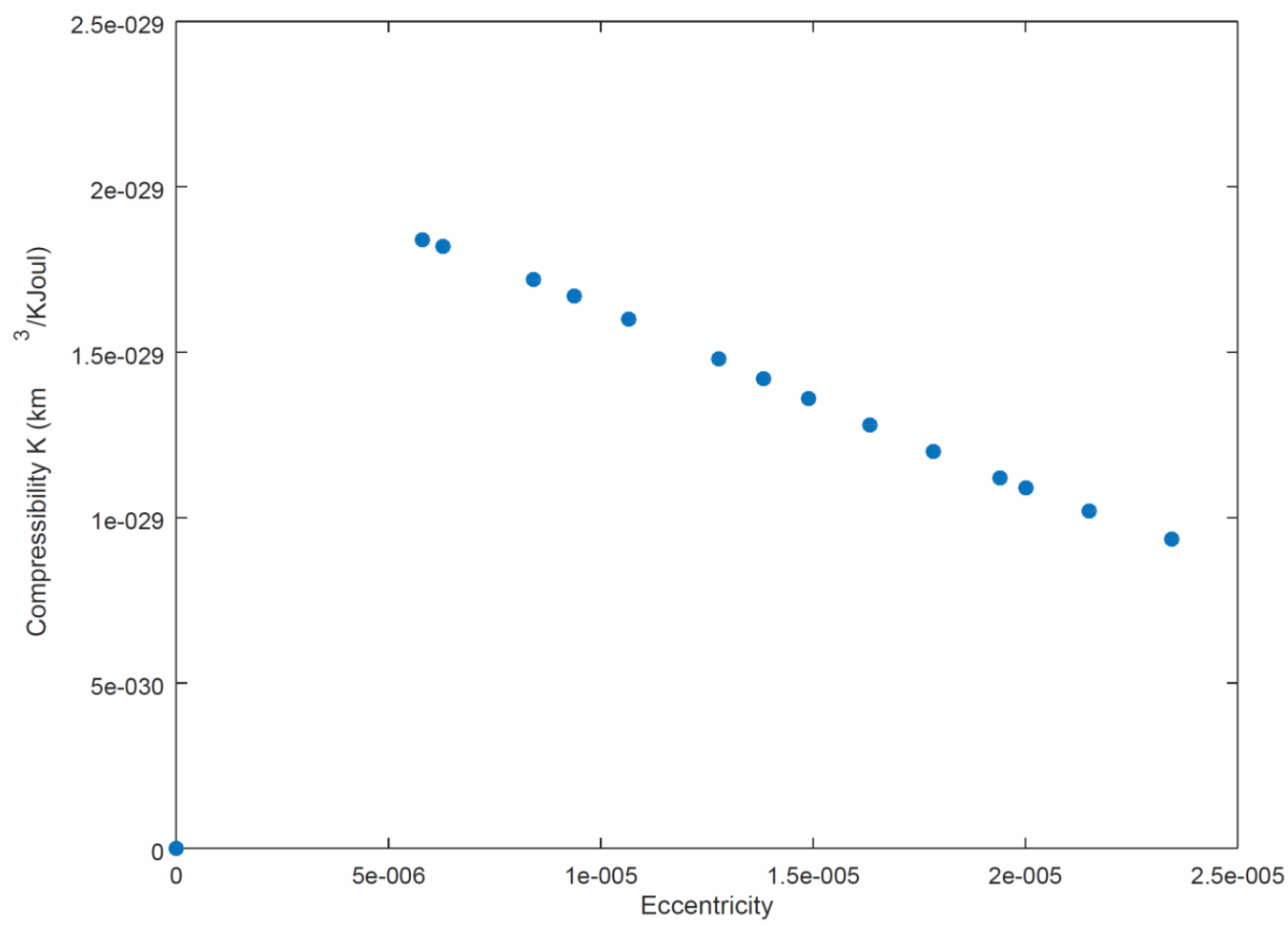

Fig. 4. The compressibility of the neutron star calculated based on the proposed model at at different eccentricities

nuclear matter are obtained for different potential models at effective masses 0.566 to 0.618 and saturation densities from 0.160 to $0.374 \mathrm{fm}^{-3}$ . The compressibility $K$ is found to be in the range 203 to $337 \mathrm{MeV}$.

The suggested model could predict each of the $B M, e, \Delta Z$ of the deformed pulsar. Based on the assumptions of the model, table 2 takes the predictions of the compressibility $K$ (in
$\mathrm{MeV}$ ), based on calculated values from M. M. Sharma [19] and K. Hassaneen and H. Mansour in reference [18], and calculates the bulk modulus $(B M)$, the eccentricity $(e)$, and the elongation along $\mathrm{z}$-axis $(\Delta Z)$. The conversions of units from $M e V$ to $\frac{\mathrm{km}^{3}}{k J o u l e}$ for $K$, requires the value of saturation nuclear density. It is taken to be $\rho_{0}=0.178 \mathrm{fm}^{-3}$, which is the average values of $\rho_{0}$ used in $[18,19]$. The conversion equation for is:

$$
\begin{aligned}
& K\left(\frac{\mathrm{km}^{3}}{\mathrm{kJoul}}\right)=\left(\frac{\mathrm{K}(\mathrm{MeV}) \times \rho_{0}\left(\mathrm{fm}^{-3}\right) \times 10^{-9} \times F_{1}}{F_{2}}\right)^{-1} \frac{\mathrm{km}^{3}}{\mathrm{kJoul}} \\
& F_{-} l=7645373 \mathrm{MeV}^{\wedge} 4 \mathrm{~V}\left(\mathrm{MeVV} / \mathrm{fm}^{\wedge} 3\right), \\
& F_{2}=5.00 \times 10^{-33} \mathrm{GeV}^{-4} /\left(\mathrm{km}^{\wedge} 3 / \mathrm{kJoul}\right)
\end{aligned}
$$

The behavior, from Table 2, of the eccentricity and $\Delta Z$ are monotonic decreasing with the compressibility $K$ of the nuclear matter in the pulsar, which shows the stiffness of the NS materials and put a restriction on the acceptable equations of states. It appears that, the estimated deformation of the pulsar due to its companion has the order of $\left(\sim 10^{-5}\right)$ for eccentricity, and the mean elongation along z-axis is of the order $10^{-9} \mathrm{~km}$. These small orders support the model assumptions. 
TABLE 2. The values of the compressibilities(K) of the neutron star material (infinite nuclear matter,) The Bulk Modulus (BM), the eccentricity (e), and the elongation along the $\mathrm{z}$-axis . The $1^{\text {st }}$ column is taken from reference $[18,19]$.

\begin{tabular}{|c|c|c|c|c|}
\hline$K(\mathrm{MeV})$ & $\left.\mathrm{K}^{\mathrm{km}} \mathrm{kmJoul}^{\mathrm{K}}\right)$ & $B M\left(\mathrm{~N} / \mathrm{km}^{2}\right)$ & Eccentricity e & $\Delta z(\mathrm{~km})$ \\
\hline 200 & $1.84 \times 10^{-29}$ & $1.087 \times 10^{29}$ & $5.798 \times 10^{-6}$ & $4.034 \times 10^{-10}$ \\
\hline 203 & $1.82 \times 10^{-29}$ & $1.099 \times 10^{29}$ & $6.283 \times 10^{-6}$ & $4.737 \times 10^{-10}$ \\
\hline 214 & $1.72 \times 10^{-29}$ & $1.163 \times 10^{29}$ & $8.415 \times 10^{-6}$ & $8.498 \times 10^{-10}$ \\
\hline 220 & $1.67 \times 10^{-29}$ & $1.198 \times 10^{29}$ & $9.375 \times 10^{-6}$ & $1.055 \times 10^{-9}$ \\
\hline 230 & $1.60 \times 10^{-29}$ & $1.25 \times 10^{29}$ & $1.066 \times 10^{-5}$ & $1.363 \times 10^{-9}$ \\
\hline 249 & $1.48 \times 10^{-29}$ & $1.351 \times 10^{29}$ & $1.278 \times 10^{-5}$ & $1.960 \times 10^{-9}$ \\
\hline 258 & $1.42 \times 10^{-29}$ & $1.408 \times 10^{29}$ & $1.383 \times 10^{-5}$ & $2.296 \times 10^{-9}$ \\
\hline 270 & $1.36 \times 10^{-29}$ & $1.471 \times 10^{29}$ & $1.489 \times 10^{-5}$ & $2.662 \times 10^{-9}$ \\
\hline 286 & $1.28 \times 10^{-29}$ & $1.563 \times 10^{29}$ & $1.634 \times 10^{-5}$ & $3.203 \times 10^{-9}$ \\
\hline 305 & $1.20 \times 10^{-29}$ & $1.667 \times 10^{29}$ & $1.783 \times 10^{-5}$ & $3.816 \times 10^{-9}$ \\
\hline 327 & $1.12 \times 10^{-29}$ & $1.786 \times 10^{29}$ & $1.940 \times 10^{-5}$ & $4.517 \times 10^{-9}$ \\
\hline 337 & $1.09 \times 10^{-29}$ & $1.835 \times 10^{29}$ & $2.001 \times 10^{-5}$ & $4.807 \times 10^{-9}$ \\
\hline 360 & $1.02 \times 10^{-29}$ & $1.961 \times 10^{29}$ & $2.150 \times 10^{-5}$ & $5.548 \times 10^{-9}$ \\
\hline 393 & $9.35 \times 10^{-30}$ & $2.139 \times 10^{29}$ & $2.345 \times 10^{-5}$ & $6.597 \times 10^{-9}$ \\
\hline
\end{tabular}

\section{Conclusions}

In the present work the authors propose a simple model to estimate the bulk deformation of static pulsar in a binary system due to the companion compact NS. The model is applied to the system PSR B1913+16, whose parameters are given in table 1, It shows a consistent behavior with the similar classical predictions from Newtonian potential as the eccentricity is diminished. The model predicts the bulk modulus $B M$ of the infinite nuclear matter and estimates the amount of the deformation. The model produces the axial elongation of the observed NS as well as the eccentricity. The eccentricity is found to be of order $10^{-5}$ and the total elongation is of the order $10^{-9} \mathrm{~km}$. The proposed model requires modification to be effectively used in pulsar data analyses. The authors will introduce the modified model in next publication.

\section{Acknowledgement}

Thanks to Physics Department, Faculty of science, Cairo University for supporting the publication of this research throughout the department workshop at 21 APR. 2018.
The last author would thank his graduation place (sebha university) for nominating me to continue the MSc. graduate studies. He thank the Libyan ministry of education for supporting him to complete his graduate studies.

\section{References}

1. Hulse, R. A., and Taylor, J. H., "Discovery of A Pulsar in Binary System", Astro. Phys. Jour., 195, (1975), L51 - L53.

2. Wolszczan, A., "A nearby $37.9 \mathrm{~ms}$ radio pulsar in a relativistic binary system", Nature, 350, (1991), 688-690.

3. Stairs, I. H., "Testing General Relativity with Pulsar Timing", Living Rev. Relativity, 6 (2003), 5-49 [Online Article].

4. Blaizot, J. P., Gogny, D., andGrammaticos, B.," NUCLEAR COMPRESSIBILITY AND MONOPOLE RESONANCES", Nucl. Phys. A265, 315 (1976).

5. Zhongyu Ma, Nguyen Van Giai, and Hiroshi Toki, "Compressibility of nuclear matter and breathing mode of finite nucleiin relativistic random phase approximation", PHYSICAL REVIEW C VOLUME 55, NUMBER 5 MAY 1997. 
6. Danielewicz, P., Lacey, R., Lynch, W. G., "Determination of the equation of state of densematter", Science, 298 (5598) (2002), 15921596.

7. Dexheimer,V. A., "Density dependent nuclear matter compressibility", PHYSICAL REVIEW C,77, (2008), 065803.

8. Horowitz, C. J., and Kai Kadau, "Breaking Strain of Neutron Star Crust and Gravitational Waves", PRL 102, (May 2009), 191102.

9. LakshmidharSatpathy, "How to go from finite nuclei to infinite nuclear matter", RIKEN Review, No. 23, (July, 1999).

10. Manchester, R. N., "Pulsars and gravity", International Journal of Modern Physics D, May 2015, Vol. 24, No. 06. This article is published also in the book "One Hundred Years of General Relativity: From Genesis and Empirical Foundations to Gravitational Waves, Cosmology and Quantum Gravity," edited by Wei-Tou Ni (World Scientific, Singapore, 2015).

11. Shriya Soma, and SarmisthaBanik ., "Deformation properties of rotating neutron stars", Proceedings of the DAE-BRNS Symp. onNucl. Phys., 60, (2015).

12. Taylor, J. H., Weisberg, J.M., "A New Test of General Relativity Gravitational Radiation and The Binary Pulsar PSR 1913+16", Astrophys. J. 253:908 (1982).

13. Thibault Damour "1974: the discovery of the first binary pulsar", Class. Quantum Grav., 32, (2015), 124009 (13pp).
14. Max Camenzind., "Compact Objects in Astrophysics, White Dwarf, Neutron Stars and Black Holes", Springer-Verlag Berlin Heidelberg,(2007).

15. John.R. Taylor, “Classical Mechanics”, University Science Books, (2005), ISBN 1-891389-22-X.

16. James.M. Lattimer, "Neutron star equation of state", New Astronomy Reviews 54 (2010), 101109.

17. James.M. Lattimer and Madappa Prakash," The Equation of State of Hot, Dense Matter and Neutron Stars", physics reports, 621 (2016), 127 164.

18. Khaled Hassaneen and HeshamMansour , " The Hot and Cold Properties of Nuclear Matter", Journal of Nuclear and Particle Physics,3(4), (2013), 77-96.

19. Sharma, M. M., "Compressibility of Nuclear Matter from Shell Effects in Nuclei", RIKEN Review, No. 23, (July 1999).

(Received:11/11/2018 accepted:21/12/2018) 


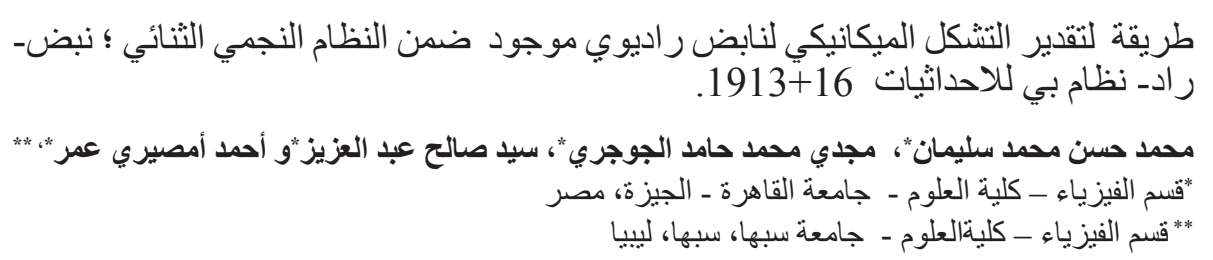

تم اعلان اكتشاف النابض الر اديوي (نبض- راد-نظام بي للاحداثيات 16+1913 ) و والمُحتوى ضمن ونظ نظام نجمي

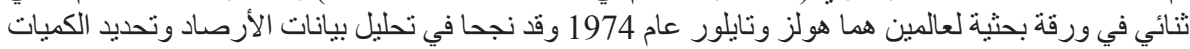

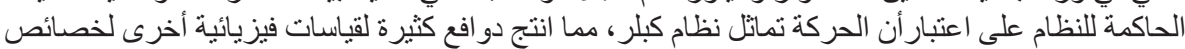

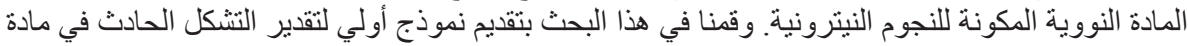

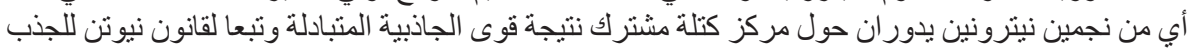

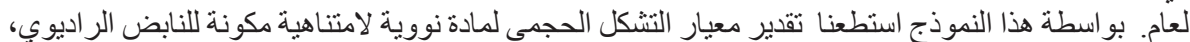

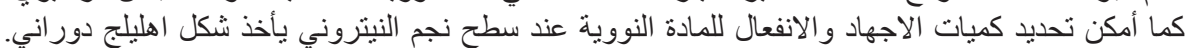

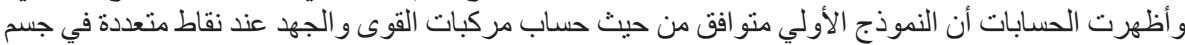

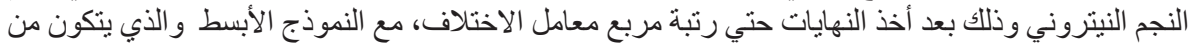

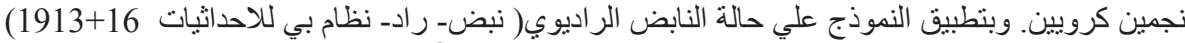

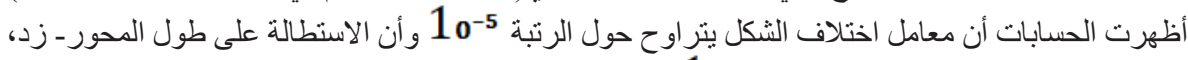

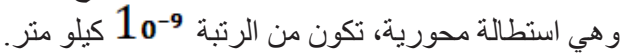

